\title{
APLICACIÓN DE LA TECNOLOGÍA LÁSER EN EL TRATAMIENTO DE LA LITIASIS URETERAL
}

José Ignacio Iglesias Prieto, Rodolfo Orozco Fariñas, Sara Gentile Nani, Jorge Massarra Halabi, José María Mancebo Gómez y Enrique Pérez-Castro Ellendt.

Servicio de Urología. Clínica La Luz. Madrid. España.

\begin{abstract}
Resumen.- OBJETIVO: Valorar las aportaciones del láser, fundamentalmente de holmio, en el tratamiento endourológico de la litiasis ureteral.

MÉTODO: Analizamos la experiencia acumulada en 154 tratamientos endourológicos con láser holmio de litiasis ubicadas a nivel ureteral, realizado de forma continuada en nuestra Unidad desde junio 2005 a diciembre 2007.

RESULTADOS: Se consiguió la fragmentación litiásica en el $100 \%$ de los casos. El uso asociado de la pinza de rama larga al láser de holmio, nos permitió alcanzar tasas de ausencia de litiasis residual en el $91 \%$ de los pacientes.
\end{abstract}

CONCLUSIONES: El tratamiento de la litiasis ureteral se ha beneficiado de los procedimientos endoscópicos, siendo el láser de holmio la fuente de energía de elección en el momento actual. La tasa de fragmentacion alcanzada fue del 100\%. Las complicaciones fueron escasas y en ningun momento de importancia.

Palabras clave: Láser de holmio. Litiasis ureteral. Láser.

Summary.- OBJECTIVES: To evaluate the contribution of laser, mainly holmium laser, to the endourological treatment of ureteral lithiasis.

METHODS: We analyze our experience in 154 endourological treatments of ureteral lithiasis with holmium laser performed in our Unit from June 2005 to December 2007.

RESULTS: Stone fragmentation was achieved in 100\% of the cases. The use of long jaw forceps associated with the holmium laser enabled us to reach a stone free rate of $91 \%$.

CONCLUSIONS: The treatment of ureteral lithiasis has benefit from the development of endoscopic procedures, being the holmium laser the energy source of choice nowadays. The fragmentation rate was $100 \%$. Complications were rare and never important.

Keywords: Holmium laser. Ureteral lithiasis. Laser. 


\section{INTRODUCCIÓN}

El tratamiento de la litiasis ureteral ha cambiado radicalmente desde la introducción y asentamiento de la Endourología, permitiendo dar una solución rápida y efectiva, que el paciente actual demanda.

Este cambio esta motivado sin duda alguna por el desarrollo tecnológico que nos ha provisto de endoscopios de menor calibre, mejor visión y mayor durabilidad convirtiéndolos en asequibles y asumibles económicamente; sin embargo los sistemas de fragmentación litiásica no se desarrollaron de forma pareja, estancándose los resultados de la técnica en cuanto a efectividad; con la llegada del láser como sistema litotritor endoscópico se produce un nuevo despegue y consolidación de la técnica.

La efectividad del proceso unido a la baja tasa de complicaciones y una correcta comercialización, han convertido al láser de holmio en el sistema de referencia actual para la fragmentación litiasica endoscópica, dejando atrás a otros sistemas de láser (pulsado de colorante, FREDDY, etc.) abandonados por su complejidad de uso y/o limitados en su tasa de fragmentación y en espera del asentamiento de otros (Erbio y Tulio) con posibilidades de alcanzar buenas tasas de efectividad con bajo riesgo asociado.

En este trabajo, revisamos nuestra experiencia con el láser de holmio en el tratamiento de la litiasis ureteral, tras haber abandonado el uso del láser pulsado de colorante, a pesar de alcanzar buenas tasas de fragmentación, por su complejo manejo y necesidad constante de mantenimiento del colorante cumarínico.

\section{MATERIAL Y MÉTODOS}

Desde el 15 de junio de 2005 a 27 de diciembre de 2007 hemos realizado 1530 tratamientos por litiasis urinaria en nuestra Unidad. Se han realizado 376 ureteroscopias por litiasis y de estas en 154 casos se procedió a la fragmentación endoluminal de la litiasis mediante láser holmio $(41 \%)$, realizando extracción con pinza de rama larga de la litiasis íntegra o fragmentada con esta, en los 222 restantes casos (59\%) (Tabla I).

Se ha llegado al correcto diagnóstico y programado el procedimiento endoscópico en base a las imágenes obtenidas mediante urografía intravenosa en el $80 \%$ de los pacientes, mientras que en el $20 \%$ restante se realizó estudio mediante radiografía asociada a ecografía, TAC o uro-RMN

El procedimiento endoscópico se ha realizado con ureteroscopios semirrígidos de Storz y Olympus $(9,8$ y 7 ,9) con canales de trabajo de 5 y $3 \mathrm{Ch}$., que permiten el paso de pinza de rama larga así como de cestillas, fibras de láser y otros tipos de aditamentos complementarios.

La colocación del paciente ha sido en todos los pacientes la ya, clásicamente comunicada (posición ginecológica con la pierna contralateral al uréter a explorar

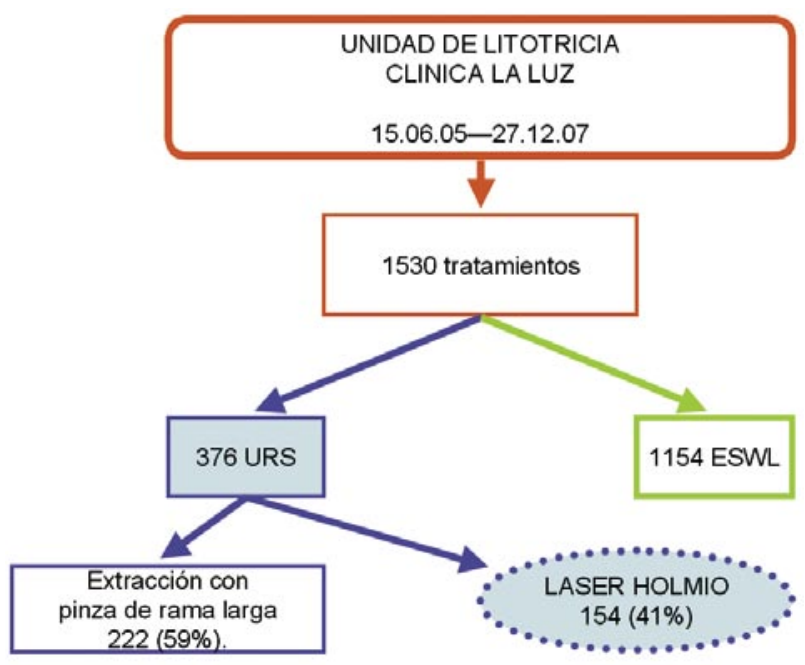

baja y en ligera rotación externa o abducción), salvo en los pacientes pediátricos en sus primeros años, en los que colocamos ambas piernas al borde de la mesa quirúrgica apoyados sobre dos rodillos de paños y en los adultos que por patología ortopédica precisaron colocación ajustada a la movilidad personal (Figura 1).
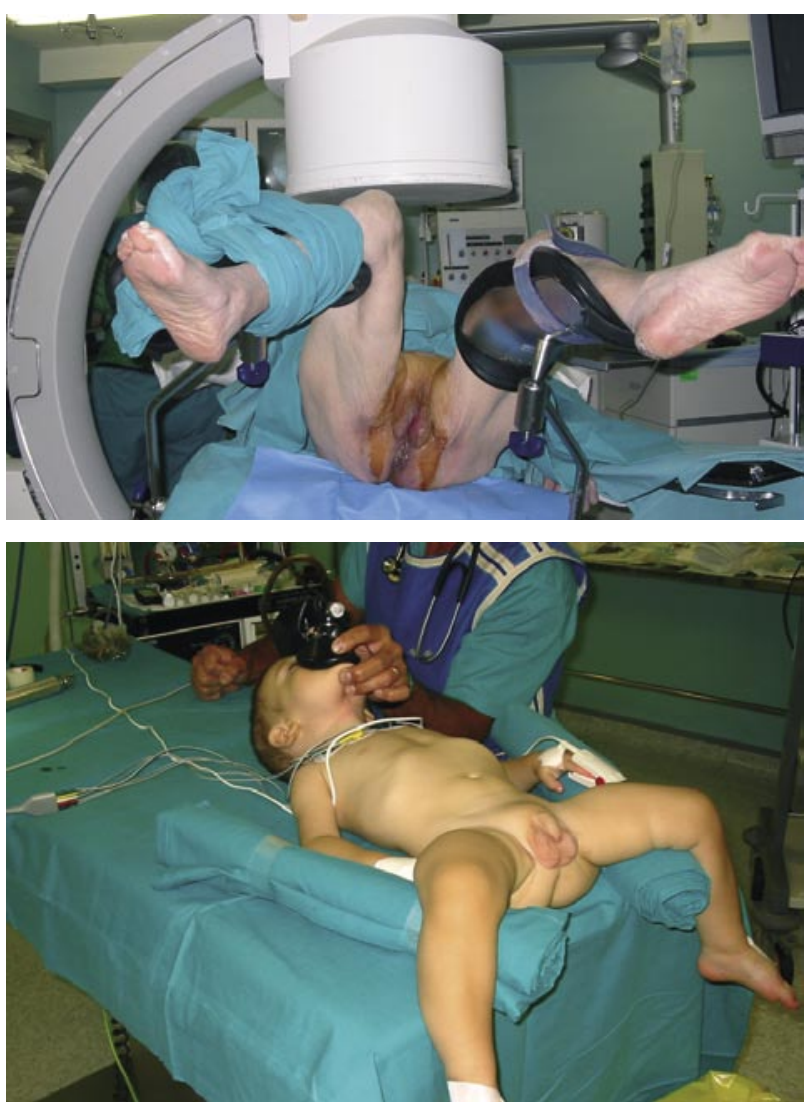

FIGURA 1. Colocación "obligada" del paciente. 
El acceso ureteral se realiza guiado mediante un catéter ureteral $5 \mathrm{Ch}$. y el uso sistemático de la bomba de infusión Ureteromat con flujo de $60 \mathrm{ml} / \mathrm{min}$. y presión de $60 \mathrm{~mm}$. Hg. (1). Ocasionalmente, cuando el meato presentaba estenosis puntiforme, se realizó dilatación del meato ureteral mediante pinza de rama larga (Figura 2). El uso de guías hidrofílicas se realizó en aquellos casos en los que el uréter yuxtavesical presentó anzuelamiento marcado y el catéter ureteral provocaba "tienda de campaña" al introducirlo en el segmento del uréter pelviano.

Hemos usado la Unidad de Láser de Holmio Medilas $\mathrm{H} 20$ de Dornier (Figura 3), que cumple con las normas establecidas por la directiva europea para productos médicos. Esta unidad genera pulsos de 350 microsegundos, con longitud de onda de $2080 \mathrm{~nm}$, frecuencia variable entre 3 y $12 \mathrm{~Hz}$. y energía máxima generada de $2500 \mathrm{~mJ}$. (potencia máxima $20 \mathrm{~W}$, conseguida con frecuencia de 8 $\mathrm{Hz}$. y energía de $2500 \mathrm{~mJ}$ o frecuencia de $10 \mathrm{~Hz}$. y energía de $2000 \mathrm{~mJ}$ ). La energía se transmitió a través de fibras ópticas de cuarzo con punta de fibra desnuda específicas para el sistema Medilas 20, de calibre de 600 micras (variable entre las $200,400,600$ y las 1000 micras) provistas de un piloto láser verde de orientación de disparo, seleccionando-siguiendo las indicaciones de Dornier- frecuencia de $8 \mathrm{~Hz}$. y energía de 0,8 J., que genera una potencia de $6,4 \mathrm{~W}$.

La energía usada osciló entre 39 y 8.986 mJ (media: 114), con un $\mathrm{n}^{\circ}$ de pulsos variable entre 35 y 11.233 (media 1317), con una duración del uso del láser oscilante entre 17 y 2423 segundos (media 184).

La edad de los pacientes tratados osciló entre los 5 años y 86 años, con una edad media de 54 años. varones.

La distribución por sexo fue de 59 mujeres y 95

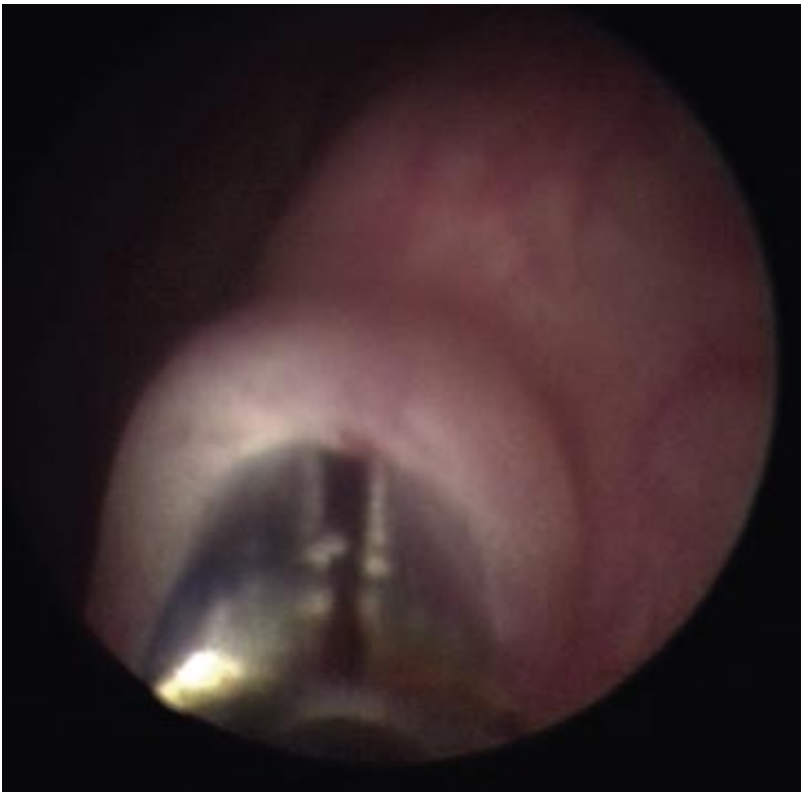

FIGURA 2. Dilatación meatal con pinza.
La localización de las litiasis fue de 12 en el tercio proximal del uréter, 25 en el tercio medio ureteral y de 117 en el tercio distal del uréter.

La lateralidad fue de 61 derecha y 93 izquierda sin diferencias entre mujer y varón (Tabla II).

El procedimiento se realizó bajo anestesia regional en 150 casos $(97,4 \%)$, y anestesia general en 4 casos $(2,6 \%)$.

La profilaxis antibiótica se realizó de forma rutinaria inmediatamente al procedimiento, mediante tobramicina $100 \mathrm{mg}$. Se continuó con ciprofloxacino 500/12 h. durante 5 días.

\section{RESULTADOS}

Se consiguió la fragmentación litiásica en el $100 \%$ de los pacientes, procediendo a completar el procedimiento mediante la extracción de los fragmentos litiásicos con pinza de rama larga en 140 casos $(90,9 \%)$, dejando al paciente libre de restos litiásicos en el mismo momento de finalizar el procedimiento. Trece casos no precisaron el uso complementario de la pinza por ser los fragmentos de mínimo tamaño. En un caso se produjo migración de frag-

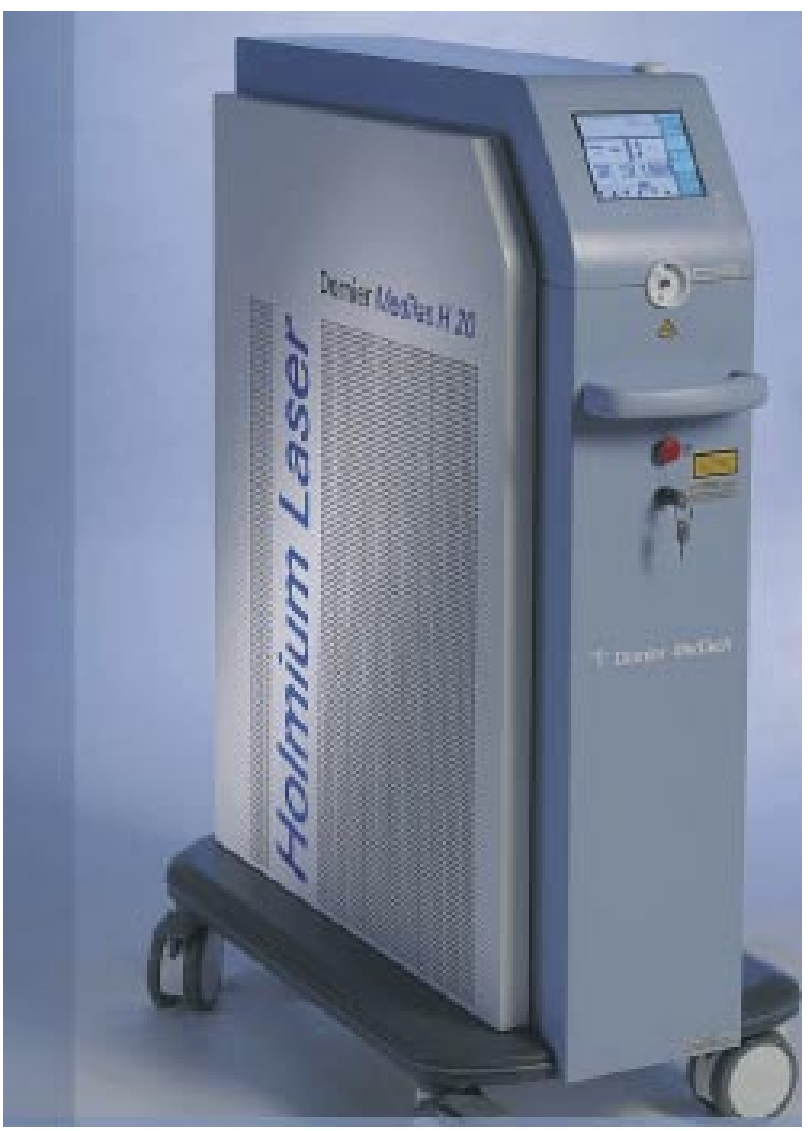

FIGURA 3. Unidad de láser. 
TABLA II.

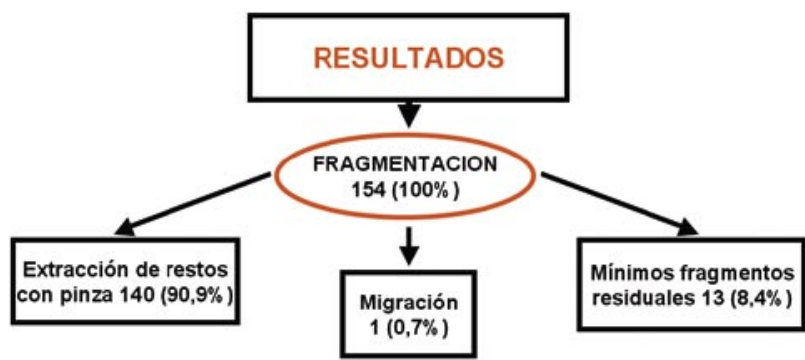

DERIVACION URINARIA POST-URS-LASER

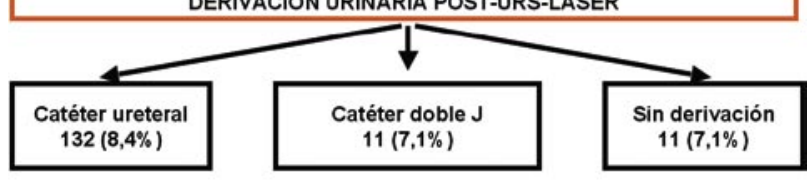

mentos litiasicos -producidos durante la fragmentación con láser- a cavidades renales, complementándose el proceso mediante litotricia extracorpórea por ondas de choque en el mismo momento.

En 6 casos se complementó el tratamiento con ESWL, por la existencia de litiasis renal asociada.

Hemos dejado colocado un catéter ureteral $5 \mathrm{Ch}$. tras el procedimiento en 132 casos $(85,7 \%)$, un catéter doble J en 11 casos $(7,1 \%)$, no dejando ningún tipo de derivación urinaria en los 11 pacientes restantes (Tabla II).

La composición de los cálculos fue en el $80 \%$ de oxalato y fosfato cálcico en sus distintas composiciones y en el $20 \%$ de acido úrico, fosfato amónico magnésico y cistina.

Como complicaciones hemos de resaltar la presencia de lesiones leves de la mucosa ureteral, provocadas durante el proceso de fragmentación y extracción de los restos litiásicos, dislaceración extensa de la mucosa en 4 casos, falsa vía en 2 ocasiones, una perforación parameatal, provocada durante el intento de acceso ureteral solucionada con cateterismo doble J, un caso de hematuria intensa-autolimitada y sin repercusión analítica- en el postoperatorio inmediato y un caso de complicación diferida con sangrado retroperitoneal autolimitado que generó un cuadro de peritonismo con resolución espontánea, lo cual supone un $5,4 \%$ de complicaciones leves y moderadas $\sin$ ninguna complicación seria, de las cuales tres de ellas (falsas vías y perforación parameatal) no están en relación al uso del láser, lo cual deja en un 3,9\% la tasa de complicaciones asociadas al uso del láser.

\section{DISCUSIÓN}

El tratamiento de la litiasis ureteral ha sufrido una completa metamorfosis en los últimos 25 años desde la introducción y asentamiento de la Endourología de forma que con el uso de la ESWL, la ureteroscopia y el abordaje percutáneo renal hayan dejado prácticamente sin opciones a la cirugía convencional con un tenue renacer en estos últimos años con el abordaje laparoscópico.

El imparable desarrollo tecnológico ha ido modificando de forma continuada los distintos modelos de ureteroscopios y sistemas de fragmentación hasta asentar al abordaje endoscópico en todos sus tramos como indicación de primera elección en el uréter medio y distal y como tercera opción en el uréter proximal (2).

En nuestra Unidad se mantienen las tasas superiores al 50\% de extracción de la litiasis sin precisar de fragmentación, incluyendo un porcentaje muy alto de litiasis de tamaño superior al centímetro de eje máximo, lo cual nos lleva a reivindicar la gran utilidad del uso conjunto del sistema de dilatación-perfusión hidráulica y la pinza de rama larga.

En el momento actual los sistemas de fragmentación endoluminal con los que se trabaja son el ultrasónico, el electro hidráulico, el neumático/electrocinético, la combinación de estos y el láser.

Aunque existen varios sistemas de láser aplicables para la fragmentación litiásica endoluminal (pulsado de colorante, Erbio:YAG, Tulio, FREDDY), el láser de Holmio se ha consolidado como referente, en los últimos años, probablemente por su versatilidad urológica y sus excelentes resultados.

En nuestra experiencia personal, abandonamos el láser pulsado de colorante cumarínico, a pesar de los buenos resultados obtenidos, debido a lo complejo de su uso (3) y tras un periodo de tiempo en el que usamos energía electrocinética, también con muy buenos resultados (4), retomamos la tecnología láser de la mano del sistema Medilas H 20 de Dornier (láser de Holmio), motivo fundamental de este trabajo.

El láser de holmio produce y libera la energía de forma pulsada, consiguiendo de esta forma minimizar las lesiones térmicas de las estructuras vecinas. La longitud de onda del láser holmio (2080nm) esta muy próxima al pico de absorción del agua (1940 nm.), situación esta que le confiere gran capacidad de fragmentación litiásica, teniendo en cuenta la gran cantidad de agua que contienen los cálculos. La fragmentación litiásica se produce mediante dos efectos sumatorios; por una parte un efecto fotomecánico que genera una onda de choque y en segundo lugar un efecto foto-térmico generado a nivel de la superficie de la litiasis, asociando así al efecto de fragmentación la capacidad de vaporizar la superficie mas externa. Este efecto foto térmico también contribuye a la fragmentación litiásica mediante la vaporización de las moléculas de agua en los cálculos formando burbujas de vapor en su interior que contribuyen a la fragmentación al expandirse.

El láser holmio genera una onda de choque relativamente débil, propiedad que disminuye la movilización de la litiasis y/o sus fragmentos (retropulsión), facilitando así el procedimiento endoscópico y disminuyendo o anulando la necesidad de usar aditamentos estabilizadores de la litiasis, confirmándolo en nuestra serie en la que en un 

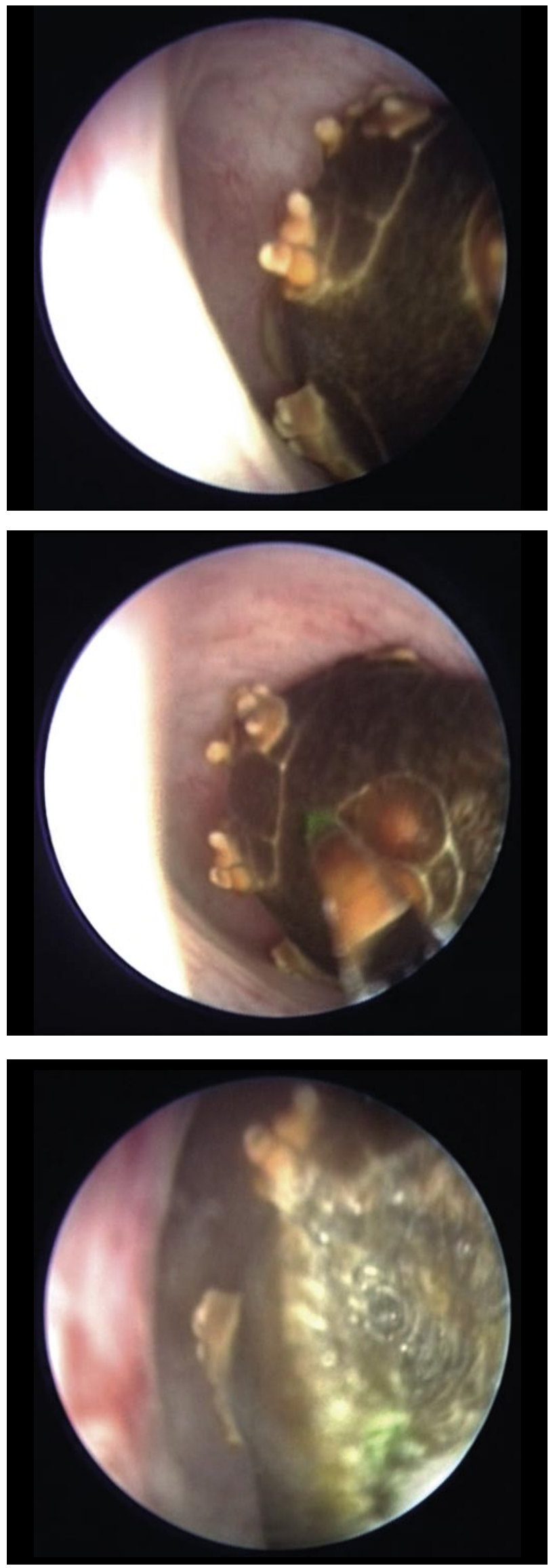

solo paciente se produjo la migración de un fragmento litiásico subsidiario de tratamiento a cavidades renales.

Debido a estos mecanismos antes expuestos la dinámica de trabajo en nuestra Unidad intenta en la medida de lo posible, huir de la fragmentación central de la litiasis, muy atractiva y vistosa, pero que posteriormente obliga a tratar cada uno de los fragmentos como a un nuevo cálculo.

Realizamos la fragmentación desde la periferia hacia el núcleo litiásico, aunque si bien es cierto que en los cálculos de mayor dureza es posible intentarlo, en los mas frágiles se provoca la fragmentación incontrolada. En los cálculos impactados invertimos la estrategia, buscando una fragmentación central rápida que nos permita desobstruir la vía y nos aleje la litiasis de la pared ureteral, habitualmente alterada con gran edema bulloso, mas lábil que en ausencia de impactación litiásica.
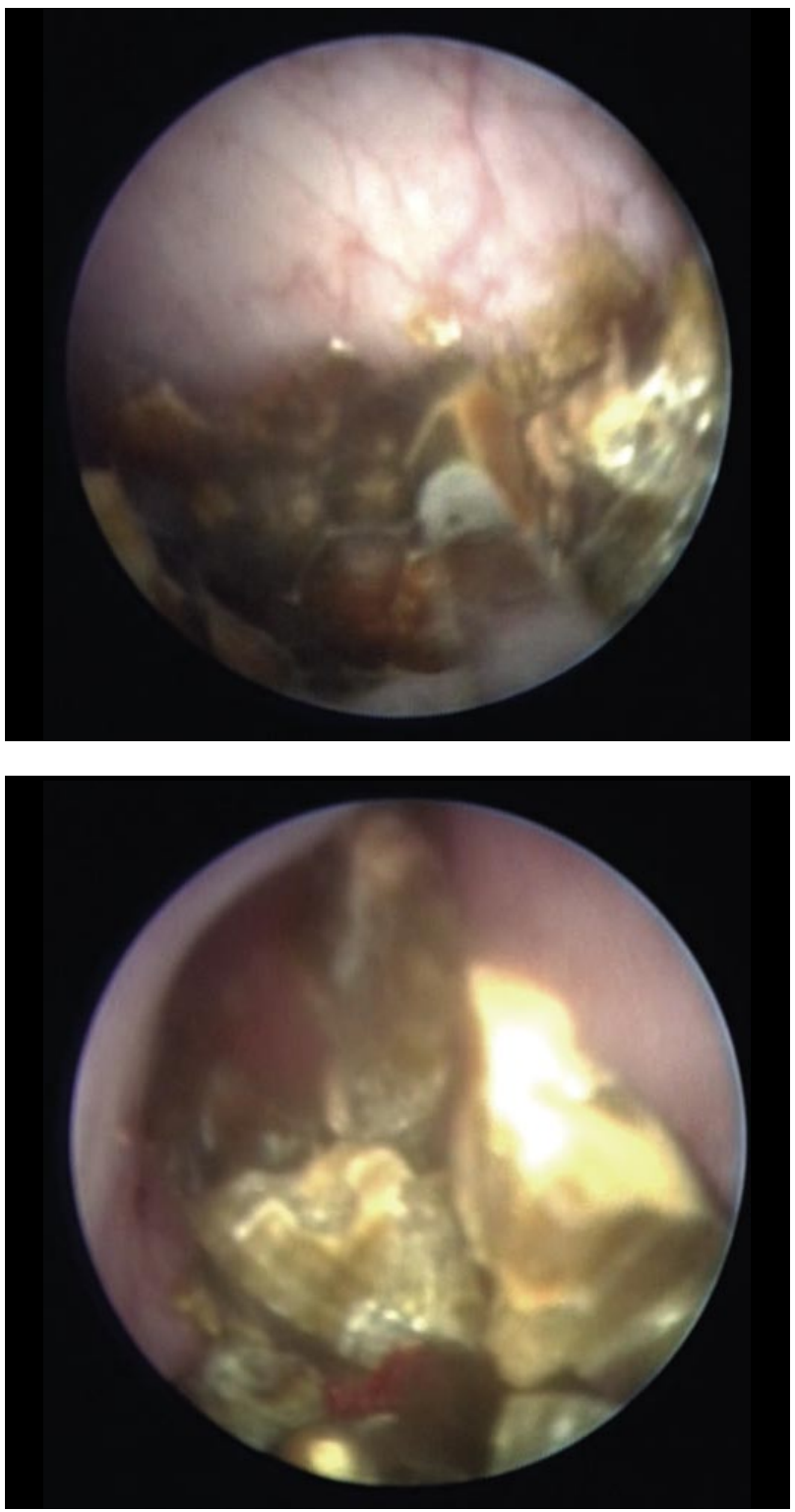

FIGURA 4. Fragmentación litiásica con láser holmio. Secuencia. 
Hemos observado la afectación de las guías hidrofílicas en su capa de recubrimiento al contacto con la fibra del láser, lo que nos hace ser muy cuidadosos durante la fragmentación cuando se trabaja con una guía en paralelo.

Como se ha comentado en el capítulo de resultados, hemos conseguido fragmentar todos los cálculos independientemente de su composición, lo cual ha evitado el uso de otros sistemas de fragmentación alternativa, situación vivida-aunque en pocos casos-en la época del uso del láser pulsado de colorante. Este excelente resultado, cien por cien de éxitos, está en la línea de los comunicados por otros grupos de trabajo que alcanzan tasas de éxito entre el 93 y $97 \%(5-7)$.

Siguiendo las recomendaciones de la casa Dornier y acorde con la experiencia de otros grupos (8), hemos realizado todos los tratamientos seleccionando una frecuencia de $8 \mathrm{~Hz}$ y una energía de $80 \mathrm{~mJ}$, lo cual genera una potencia de 6,4 W. Llama la atención el margen tan amplio de energía sobrante del equipo -potencia máxima $20 \mathrm{~W}$ - motivo por el que en el momento actual hemos solicitado mas datos técnicos de cara a la modificación de los parámetros de frecuencia y energía que nos permitan disminuir el tiempo de tratamiento.

El uso de energías superiores a 1 Julio provoca la degradación rápida de la fibra (8); en nuestra experiencia hemos encontrado una degradación muy rápida de la fibras cuando trabajamos en la exéresis de tejido blando (neoformaciones uroteliales) a pesar de no modificar los parámetros de frecuencia y energia usados para la fragmentación litiásica

Se ha descrito la producción de cianuro durante la fragmentación de cálculos de ácido úrico (9), lo cual hace que la fragmentación de grandes litiasis de ácido úrico deban ser consideradas como de riesgo si se asocian a lesiones mucosas que faciliten la absorción sanguínea.

La lesión provocada por efecto térmico del láser se prolonga a los $4 \mathrm{~mm}$. en profundidad de la zona de contacto de la fibra, lo cual hace que las lesiones sean mínimas, teniendo en cuenta que la distancia de aplicación supera habitualmente los $5 \mathrm{~mm}$. desde la superficie litiásica, aun así en las zonas de proximidad de estructuras vasculares se deben extremar las precauciones. Otra precaución a tomar si se opta por "perforar" el cálculo, es tener un control estricto y muy frecuente de la fragmentación a fin de evitar la tunelización completa de la litiasis y lesionar la pared ureteral.

Hemos tenido la oportunidad de usar la unidad de láser de holmio de Storz, con la que hemos obtenido resultados similares, encontrado más dificultad en orientar la fibra hacia el punto deseado debido al color del piloto, rojo en este caso, mas difícil de visualizar en un medio turbio.

En nuestra serie no hemos tenido lesiones ureterales importantes salvo la existencia de esfacelos en las zonas de tratamiento de las litiasis enclavadas.
Dentro de las complicaciones merece mención aparte el cuadro presentado por una paciente en la que tras realizar tratamiento de forma rutinaria de un cálculo a nivel pelviano, inicio a las $24 \mathrm{~h}$. con un cuadro de peritonismo y en el que se documentó una colección hemática retroperitoneal, que por exclusión de otras patologías se adjudicó a lesión vascular adyacente por injuria térmica secundaria al uso del láser. El cuadro fue autolimitado y se resolvió de forma espontánea. Este cuadro recuerda a otro referido en la literatura en el que aparece hematuria severa diferida en el tiempo, con necesidad de transfusión y embolización de fístula arteriovenosa, tras la fragmentación de litiasis en el cáliz inferior renal mediante cirugía intrarenal retrógrada y uso del láser de holmio (10).

Realizamos en este estudio los tratamientos mediante ureteroscopios rígidos motivados por la mayor experiencia de manejo, su mayor versatilidad en cuanto al uso de sistemas de extracción (pinza, cestilla, etc.) y su economía. La aparición de camisas de acceso ureteral permite el uso de ureteroscopios flexibles de forma más frecuente, disminuyendo las roturas al permitir una navegación mas sencilla y en el caso de camisas con doble sistema el uso independiente de aditamentos que evitan la perforación del canal de trabajo. Pensamos que en futuro el uso del ureteroscopio flexible modificará el algoritmo del tratamiento de la litiasis del uréter proximal hasta convertirla de elección. De forma sistemática procedemos a la colocación de un catéter ureteral no5 $\mathrm{Ch}$. hasta el día siguiente en el momento del alta sanatorial, y solamente se deja un catéter doble J en los casos en los que existía intenso edema perilitiásico o se provocó lesión mucosa durante la aplicación del láser y en los casos en los que existe litiasis renal ipsilateral subsidiaria de tratamiento con ESWL y que por su tamaño aconsejen la colocación previa de catéter doble J.

Existe en el momento actual- con discrepanciasuna tendencia mundial a no dejar sistemas de derivación post-ureteroscopia (11); en nuestro grupo defendemos la abstención de colocar de forma sistemática derivación con doble J, sin embargo la experiencia hace que nos decantemos por dejar una catéter ureteral convencional $5 \mathrm{Ch}$. (el mismo que usamos para guiarnos en la introducción del ureteroscopio) durante unas horas, más si tenemos en cuenta que realizamos el procedimiento, en la mayoría de los casos, con el paciente ingresado y bajo anestesia regional que nos obliga a dejar sonda vesical; tenemos la impresión- no documentada- de necesitar menos analgesia en los pacientes en los que se deja catéter ureteral que disminuye el edema del segmento ureteral en contacto con la litiasis.

Existen otros sistemas láser para la fragmentación litiásica, que constituyen otras alternativas. El láser FREDDY (frecuency doubled doubled-pulse $\mathrm{Nd}$ :Yag) es uno de los primeros sistemas láser usados en la fragmentación litiási$\mathrm{ca}$, es un láser pulsado que provoca la fragmentación litiásica mediante un efecto fotomecánico, presenta un perfil de seguridad muy alto, pero no consigue la fragmentación de cálculos duros (oxalato cálcico monohidrato, cistina, bruxita) motivo por el que su uso ha decaído a favor de otros tipos de láser. El láser de Erbio presenta características muy similares a las del láser Holmio con una penetración en profundidad mucho menor que este, lo cual le confiere la 
posibilidad de ser usado en los mismos procedimientos con mayor seguridad respecto a los tejidos blandos adyacentes.

El láser de Tulio es un sistema adaptable a las necesidades pudiendo usarse como pulsado para el tratamiento de la litiasis o como continuo para la ablación de tejido blando; la fragmentación litiásica se consigue unicamente mediante efecto foto térmico lo cual incrementa la posibilidad de lesiones térmicas adyacentes. De estos dos últimos tipos de láser desconocemos la comercialización en nuestro país.

\section{CONCLUSIONES}

El tratamiento de la litiasis ureteral se ha beneficiado de los procedimientos endoscópicos, de forma que estos se convierten en electivos en los segmentos distal y medio y como una posibilidad en el segmento proximal.

El láser de Holmio ha contribuido de forma importantísima en la consecución de este hecho al posibilitar de forma efectiva y segura la fragmentación litiásica a través de endoscopios de bajo calibre tanto rígidos como flexibles.

El uso de camisas de inserción y protección de los ureteroscopios flexibles posibilitan que el uso de estos sea mas frecuente al facilitar la inserción y navegación del ureteroscopio y sobre todo al prolongar la vida del endoscopio abaratando el coste por procedimiento.

La tasa de éxitos en la fragmentación litiásica en nuestra serie es del $100 \%$, con un $91 \%$ de pacientes libres de litiasis en el mismo proceso al asociar la extracción con pinza de los fragmentos generados.

El número de complicaciones generadas por el uso del láser es mínimo, y en ningún caso ha sido una complicación grave, aun así y en base a la sospecha de posible iatrogenia térmica se deben extremar las precauciones durante su uso en zonas próximas a los vasos.

La dinámica de tratamiento debe huir de la tunelización y fragmentación central de la litiasis excepto en los casos de enclavamiento.
Consideramos que la fragmentación litiásica ureteral mediante láser de Holmio es actualmente en nuestro medio, la mas efectiva y segura, siendo para nosotros el método de elección en su uso endourológico.

\section{BIBLIOGRAFÍA y LECTURAS RECOMENDADAS}

1. CIMA, L.; IGLESIAS PRIETO, J.I.; OROZCO FARIÑAS, R. y cols.: "Dilatación hidráulica (Uretoromat): Ureteroscopia eficaz y segura". Arch. Esp. Urol., 61: $55,2008$.

2. TISELIUS, H. G.; ACKERMANN, D.; ALKEN, P. et al.: "Urolitiasis". Eur. Urol., 40: 362, 2001.

3. IGLESIAS PRIETO, J.I.; MANCEBO, J.M.; MASSARRA, J. y cols.: "Tratamiento de la litiasis ureterál con láser”. Arch. Esp. Urol., 45: 33, 1992.

4. FELDMAN, D.E.; IGLESIAS, J.I.; AGUADO BLASS, C. y cols.: "Láser pulsado de colorante vs litotritor EKL-Compact en el tratamiento, litofragmentador de la litiasis ureteral distal". Arch. Esp. Urol., 53: 357, 2000.

5. SOFER, M.; WATTERSON, J.D.; WOLLIN, T.A. et al.: "Holmium: YAG láser lithotripsy for upper urbi tract calculi in 598 patients". J. Urol., 167: 31, 2002.

6. GRASSO, M.: "Experience with the Holmium láser as an endosCopie lithotrite". Urology, 48: 199, 1996.

7. PALMERO MARTÍ, A.; BUDÍA ALBA, J.L.; PONTONES MORENO, E. y cols.: "Tratamiento endoscópico de la litiasis pieloureteral mediante láser HolmiumYAG. Dos años de experiencia”. Actas Urol. Esp., 29: 587, 2005.

8. PIERRE, S.; PREMINGER, G.M.: "Holmium láser for stone management". World J. Urol., 25: 235, 2007.

9. TEICHMAN, J.M. et al.: "Holmium: YAG Litohitripsy of uric acid calculi". J. Urol., 160: 2130, 1998.

10. TIPLITSKY, M.D.; MILHOUA, M.D.; MANO, B et al.: "Intrarenal arteriovenous fistula after ureteroscopic stone extraction with Holmium Láser Lithotripsy". Journal of Endourology, 21: 5, 2007.

11. DJALADAT, H.; TAJIK, P.; PAYAN DE MENR, P.; et al.: "Ureteral catheterization in uncomplicated ureterolithotripsy: A randomized, controlled trial". European Urology, 52: 836, 2007. 